\title{
Coordination Need Not Be A Problem
}

\author{
Prajit K. Dutta* \\ First Draft: March 8, 1999; Current Version: March 24, $2003^{\dagger}$
}

\begin{abstract}
In a game of common interest there is one action vector that all players prefer to every other. Yet there may be multiple Pareto-ranked Nash equilibria in the game and the "coordination problem" refers to the fact that rational equilibrium play canot rule out Pareto-dominated equilibria. In this paper, I prove that two elements - asynchronicity and a finite horizon - are sufficient to uniquely select the Pareto-dominant action vector (in subgame perfect equilibrium play).
\end{abstract}

\section{Introduction}

In a common interest game there is one action vector that all players prefer to every other action vector. There may however be multiple Pareto-ranked Nash equilibria in the game. The "coordination problem" refers to the fact that rational equilibrium play canot rule out these other Pareto-dominated equilibria.

The principal contribution of this paper is to show that coordination is not a problem when a common interest game is played over a long but finite horizon provided players asynchronize their moves. More generally, coordination is not a problem if players have the option to asynchronize

\footnotetext{
${ }^{*}$ Department of Economics, Columbia University, New York, NY 10025; pkd1@columbia.edu.

${ }^{\dagger}$ The paper has benefitted from the comments of seminar participants at Rice, Columbia, Texas A\&M, ISI, New Delhi, Georgetown, Florida International, and the International Game Theory Conference at SUNY Stonybrook. An earlier version of the paper was significantly improved by the comments of Drew Fudenberg, Glenn Ellison, John Van Hucyk, Bob Rosenthal and Rajiv Sethi while the present version has been discussed with Roger Lagunoff, Paul Milgrom and Roy Radner. Unfortunately, I still bear sole responsibility for all errors.
} 
moves. In either case there is a unique equilibrium outcome and it is one in which the Pareto-dominant action gets played for all but a uniformly bounded number of initial periods.

A secondary contribution of the paper is to introduce an endogenous model of timing, a model in which players decide when they want to move, a model that nests the standard simultaneous move model as well as the alternating move model.

Common interest games have been extensively studied in recent times and have been used to explain a variety of phenomena, including the Great Depression, bank runs, credit cycles, the Poverty Trap in developing economies, and the speed of adoption of new technologies in the presence of network externalities. See, for example, the analysis and detailed references in the book by Cooper (1999) - and for references in Industrial Organization, Tirole (1989). One special case - and one studied in many of these applications - is pure coordination where players agree on the entire preference order and not just on the best element. Note that repeating the common interest stagegame, and applying subgame perfection as the equilibrium criterion, makes the coordination problem even a little worse because a variety of behavior are supportable as equilibria (thanks to the folk theorems of Benoit-Krishna (1985) and Fudenberg-Maskin (1986)). ${ }^{1}$

An asynchronous game is one in which at any period $t$ at most one player gets to change her action (and those of the others remain fixed at their last action choice). Payoffs are, however, ongoing: the period $t$ payoff vector depends on the action that was selected by the mover in that period as well as on the fixed actions of the others. A leading example of an asynchronous game is a game of alternating moves. ${ }^{2}$ Asynchronicity often reflects technological or economic constraints such as time to build or costs to changing the status quo.

In an asynchronous game, as also in a simultaneous move game, the timing of moves is exogenously specified. A second contribution of this

\footnotetext{
${ }^{1}$ Experimental evidence is mixed on whether players in "real-life" always play the Pareto-dominant action vector. See, for example, Cooper et. al (1990) and Van Huyck et. al (1990). Also see Section 6 for a comment on the experimental literature in light of the results in this paper.

${ }^{2}$ There is a growing literature on alternating move games. In Industrial Organization theory there is the important paper pair of Maskin-Tirole (1988 a,b), a much earlier treatment by Cyert-DeGroot (1970) and a more recent one of Wallner (1999). Alternating move pure coordination games have been recently studied by Lagunoff-Matsui (1998) and private provision of public goods by Gale (1995). More general treatments may be found in Friedman (1988), Bhaskar-Vega-Redondo (1998), and Wen (1998).
} 
paper is to endogenize that timing. An asynchronisable game is one in which players can choose when to move. Within some constraints to be detailed in Section 2, players can choose any pattern of moves - they can move simultaneously or in an alternating sequence or some intermediate hybrid pattern.

I consider finite horizon games and use backward induction to study the set of subgame perfect equilibria of common interest games. Two main results are proved.

The first result is for asynchronous games (and, by corollary, for alternating move games). In that case there is a uniform upper bound, say $\Gamma$, within which every subgame perfect equilibrium converges to the Pareto-dominant action vector of the stage game (and is absorbed thereafter). Moreover the bound is independent of the horizon: hence, whenever the horizon is long, there is no coordination problem except in the first (few) periods. In particular, the unique limiting average equilibrium payoff is the Pareto-dominant payoff. $^{3}$

This contrasts with results from finite horizon simultaneous play of a common interest game. In such games, the constant play of a Paretodominated stage equilibrium is a subgame perfect equilibrium - and for long horizons, every individually rational payoff is an equilibrium payoff. And that brings up the question: which of these two dramatically different conclusions is more robust? That of simultaneous moves or that of asynchronous/alternating moves?

I study that question within the structure of asynchronisable games. The option of asynchronising moves has a striking implication: again equilibria are always efficient - there is a maximal number of periods of inefficient play in any subgame perfect equilibrium. Yet again the bound is uniform; $\Gamma$ is independent of the horizon (and initial level of miscoordination). In particular, even if the players are initially stuck in a simultaneous move inefficient equilibrium they will transit to Pareto-dominant play within the maximal number of periods.

In terms of the literature, the two papers closest to the current one are Lagunoff-Matsui (1997) and Gale (1995). Lagunoff-Matsui (1997) studied infinite horizon alternating move pure coordination games and showed that the unique equilibrium outcome for high discount factors is the Pareto-

\footnotetext{
${ }^{3}$ The same bound $\Gamma$ also applies for all initial level of miscoordination, i.e., it does not matter how many players are playing inefficiently to begin with or indeed what they are playing.
} 
dominant one. ${ }^{4} \quad$ Gale (1995) studied public provision of private goods and proved an efficiency result. Although the model in that paper is quite different from the current one, the induction method of proof is similar. ${ }^{5}$ For a further discussion of the two papers, please see Section 6.1.

In terms of the remaining literature, some authors have suggested augmenting the game structure. There have been appeals to bounded rationality - memory bounds in the case of Aumann-Sorin (1989) or computability restrictions in the case of Anderlini (1991) - or to pre-play communication as in the case of Matsui (1991). A common lesson from all these papers is that one needs surprisingly strong assumptions to rule out Pareto-dominated equilibria.

The two main results of this paper show that two oft-used elements (potential) asynchronicity and a finite horizon - are together sufficient to uniquely select the Pareto-dominant action vector. The two elements are also minimally necessary since without either one there is a folk theorem in common interest games. Benoit-Krishna (1985) prove that for simultaneous move finite horizon games (Benoit-Krishna 1985). There is also a folk theorem for asynchronous infinite horizon games (Dutta 1996). ${ }^{6}$

The finiteness of horizon allows us to employ backward induction and that has an important implication for asynchronous common interest games: if somehow, sometime, players converge to the Pareto-dominant action vector, then they stay there for the remaining periods. That players have the option to asynchronise then plays a critical role in a second (and lengthy!) argument. This argument shows that in asynchronous and asynchronisable games, a sequence of players must build play up to the absorbing Paretodominant state.

To summarize, in the standard simultaneous move model players are forced to synchronise their move times. And that seemingly innocent assumption makes all the difference in the world. When players have the option to asynchronise - or are required to do so - coordination is not a problem.

\footnotetext{
${ }^{4}$ The 1997 paper applied to pure coordination games. Recently, in Lagunoff-Matsui (2002), they have partially extended the result to more general stage-games.

${ }^{5}$ An important feature of the model is irreversibility in actions. Gale (2000) studies a more general model of irreversibility.

${ }^{6}$ Dutta (1996) proved a folk theorem for infinite horizon stochastic games. Stochastic games, as is well-known, are like repeated games except with state variables. An asynchronous move game has a state variable which is the fixed action of players other than the current mover.
} 
The model is discussed in Section 2. Section 3 presents the basic results for the alternating moves case while Section 4 generalizes the results to allow for endogenous asynchronisable moves. Section 5 discusses some other results and extensions and Section 6 concludes with a discussion of policy implications and the relevant literature. Some of the longer proofs are collected in section 7 .

\section{$2 \quad$ Model}

In this section, I present two models - an asynchronous game and an asynchronisable game. But first I will discuss in some detail common interest stage games.

\subsection{Common Interest Stage Game}

Let $G$ denote an $N$ player stage game (in strategic form). Denote player $i$ 's strategy set $A_{i}$ and her payoff function $\pi_{i}$, where, as usual, $\pi_{i}: A \rightarrow \Re$, and $A \equiv A_{1} \times A_{2} \times A_{3} \times \ldots A_{N}$ is the set of strategy tuples for the $N$ players. Suppose that $A_{i}$ is finite for every $i$.

Definition The stage game $G$ is said to be a game of common interest if there is a strategy tuple that gives each player her highest payoff in the game, i.e., if $\exists \widehat{a} \in A$ such that $\pi_{i}(\widehat{a})>\pi_{i}(a)$ for all $a \neq \widehat{a}$ and for all players $i=$ $1,2, \ldots . N$.

A few examples will help clarify the definition.

Example 1 Pure Coordination Game - common preference order ${ }^{7}$

$$
\begin{array}{ccc}
1 \backslash 2 & \widehat{a}_{2} & \widetilde{a}_{2} \\
\widehat{a}_{1} & 1,1 & -1,-1 \\
\widetilde{a}_{1} & -1,-1 & 0,0
\end{array}
$$

Example 2 General Coordination Game - common best element

$$
\begin{array}{ccc}
1 \backslash 2 & \widehat{a}_{2} & \widetilde{a}_{2} \\
\widehat{a}_{1} & 1,1 & -100,-1 \\
\widetilde{a}_{1} & -1,-100 & 0,0
\end{array}
$$

Example 2 satisfies a property called strategic complementarity; the increment $\pi_{i}(\widehat{a})-\pi_{i}\left(\widetilde{a}_{i}, \widehat{a}_{j}\right)$ - when player $j$ plays the efficient action $\widehat{a}_{j}$ - is

\footnotetext{
${ }^{7}$ In a more general version of a pure coordination game, players agree on the ranking over the payoff numbers $\pi_{i}(a)$ although their payoffs are not necessarily identical.
} 
larger than the corresponding increment $\pi_{i}\left(\widehat{a}_{i}, \widetilde{a}_{j}\right)-\pi_{i}(\widetilde{a})$ when $j$ plays the inefficient action. ${ }^{8}$ Not all common interest games satisfy strategic complementarity:

Example 3 Common Interest Game - common best element but not strategic complementarity

$$
\begin{array}{lccl}
1 \backslash 2 & \widehat{a}_{2} & \widetilde{a}_{2} & a_{2}^{\prime} \\
\widehat{a}_{1} & 1,1 & -100,-1 & 0,0 \\
\widetilde{a}_{1} & -1,-100 & 0,0 & -200,-200
\end{array}
$$

Here is a (simple) economic example of a common interest game.

\section{Example 4 Partnership/Public Provision}

Suppose that the $N$ players jointly provide some input into a production process that has the following indivisibility: if the action vector chosen is $\widehat{a}$ $=\widehat{a}_{1}, \widehat{a}_{2} \ldots \widehat{a}_{N}$, then and only then is output produced. Suppose the payoff to player $i$ - when output is produced - is equal to $\pi_{i}-d_{i}(\widehat{a})$ while the payoff to any other action vector $a^{\prime}$ is $-d_{i}\left(a^{\prime}\right)$, where $d_{i}($.$) is (like) a disutility of$ effort function. The stage game has common interest if $\pi_{i}>d_{i}(\widehat{a})-d_{i}\left(a^{\prime}\right)$ for all other action vectors $a^{\prime}$.

Note that in each of the examples there are two pure strategy Nash equilibria of the stage game: $\left(\widehat{a}_{1}, \widehat{a}_{2}\right)$ and $\left(\widetilde{a}_{1}, \widetilde{a}_{2}\right) \cdot{ }^{9}$

\subsection{Model 1 - (Exogenous) Asynchronous Game}

Definition: An asynchronous game (with horizon $T$ and stage game $G$ ) is described by a player assignment function $X:\{1,2, . . T\} \longrightarrow\{1,2$, .. $N\}$. At time $t$, player $X(t)$ - and only that player - gets to move.

The fact that the assignment function is part of the game's data is the exogeneous part of the definition while the fact that only one player gets to move in any period is the asynchronous part. ${ }^{10}$

\footnotetext{
${ }^{8}$ In the literature, there are multiple terms for the same class of games. Aumann-Sorin (1989) call a game that has a Pareto-dominant action vector, i.e., satisfies the definition above, a game of common interest. I follow their terminology. Cooper (1999) - and some other authors - call such games coordination games. However, the latter authors also often impose strategic complementarity (and certain other conditions) in order to derive their results. Consequently I will reserve the term coordination game for common interest games that additionally satisfy strategic complementarity.

${ }^{9}$ In Example 4, the $\widetilde{a}$ vector is an equilibrium if for every player $d_{i}(\widetilde{a}) \leq d_{i}\left(a_{i}, \widetilde{a}_{-i}\right)$ for all $a_{i}$.

${ }^{10}$ The analysis can be easily extended to the case where there may not be a mover in certain periods.
} 
Whenever it is player $i^{\prime} s$ turn to move, he can choose any action $a_{i t}$ from the set of feasible actions in the stage game, $A_{i}$ - and this action then remains fixed till the next time $i$ can change his action. Furthermore, payoffs are ongoing, i.e., each player gets a payoff in every period $t$. If $\bar{a}_{-i t}$ denotes the (fixed) action of all players other than the mover at time $t$, then the payoff to player $j$ in that period is given by $\pi_{j}\left(a_{i t}, \bar{a}_{-i t}\right), j=1, \ldots N$.

The leading example of asynchronous games are alternating move games in which the $N$ players move sequentially, starting with player 1 . Hence, player $i$ gets to first move in period $i$ and then moves again in periods $m N+i$, for $m=1,2,$. In this paper, I will discuss alternating move games in detail and subsequently show in Section 5 that all the results hold for arbitrary asynchronous games as well.

Since payoffs are ongoing, every asynchronous game needs a specification of an initial action state $a \equiv a_{1}, \ldots a_{N}$ for the game. Every action in that initial state remains fixed in period 1 except the action choice of the period 1 mover. And so on. I will consider all possible initial action states.

\subsection{Model 2 - (Endogenous) Asynchronisable Game}

The more general model is one of endogenous timing in which players decide a) when to move and b) whether or not to move asynchronously. The choice of when to move will be modelled as follows: if a player chooses to next move $\zeta$ periods from today then she cannot change her action for the next $\zeta-1$ periods. As regards b), a player can asynchronise her moves from those of the other players in the following sense: once a player commits to next move $\zeta$ periods from today, future movers cannot choose that exact same date as their time of move. Needless to add, other current movers may also choose to next move the same number of $\zeta$ periods from today.

In other words, whenever it is her time to move, a player does two things - she chooses her next move-time, say, $t+\zeta$, and she chooses an action that she plays in the current period $t$ and that she commits to for periods $t+1$, $t+2, \ldots t+\zeta-1 .^{11}$ However that date can be chosen only if $t+\zeta$ was not already picked as the next time of move by some player(s) in a period before $t$. I will further impose the restriction that the longest period of commitment is $N$, the number of players in the game. ${ }^{12}$

\footnotetext{
${ }^{11}$ Of course if $t+\zeta=t+1$, then she is not really committed to any action since she gets to make an action choice in the very next period.

${ }^{12}$ I impose the restriction that the longest period of commitment is $N$ for two reasons - one, that is indeed the case with alternating moves and second to make the analysis
} 
Let us consider some examples:

Example 1 Simultaneous Moves - Initially and Forever: Every player has a move at time $t$ and every player picks $t+1$ as the next time of move. Futhermore, the same timing choices are made every period.

Example 2 Simultaneous Moves Transiting to Alternating Moves: Every player has a move at time $t$ (and so we begin with simultaneous moves and all future dates are available to be chosen). In period $t$, player 1 picks $t+\zeta=t+1$, player 2 picks $t+\zeta=t+2$, and so on through player $N$ who picks $t+\zeta=t+N$. Thereafter, the players are locked into the alternating move structure.

More formally, we have:

Definition: $A$ date $t+\zeta$ is said to be available at $t$ if no player chose it as a time of next move in any of the periods $t-1, t-2, \ldots t-N+1$. Let $\Theta_{t}$ be the set of dates available at $t$.

Definition: A time $t$ strategy for any mover, say player $i$, is given by a pair $\left(a_{i t}, \theta_{i t}\right)$ where $a_{i t}$ is an element from the set $A_{i}$ and $\theta_{i t}$, the date of next move, is an element of $\Theta_{t}$.

Remark 1: An attempt to asynchronize may not succeed. For instance, when there are multiple current movers, simultaneously some other player might also pick the same currently available date for their next move.

Remark 2: In this model, once a player asynchronizes she is forever asynchronized; thereafter, no one else can ever choose to move simultaneously with her nor can she choose to move simultaneously with the other players. There is a more general model in which asynchronization might be temporary; after some "cycles" in which $i$ moves by herself, she might choose to move with other players (and vice-versa). The key idea in that general model is that deciding at date $t$ to move again at date $t+1$ is quite different from deciding to move next at a date strictly greater than $t+1$; whereas the latter involves a commitment to hold a constant action for a while there is no such commitment in the former case. Formally, in the general model a player can choose to move either at date $t+1$ or at any available date thereafter.

One consequence of this expanded choice is that an alternating move structure is no longer an absorbing state. It will be shown in Section 5.3 that the main theorem continues to hold for this general model. (Needless to add, the proof is more cumbersome.)

interesting. The reader can convince himself that Pareto optimality is more likely to emerge if players are allowed to make long commitments to the Pareto-optimal action. 
As with the exogenous move model, each player gets a payoff in every period based on the actions chosen by the current mover(s) and the fixed actions - if any - of the other player(s). Since payoffs are ongoing we have to specify an initial state in this model as well. Again, I will be completely agnostic and allow every possible initial state.

\subsection{Finite Horizon}

I study finite horizon games that end after period $T(<\infty)$. Lifetime payoffs are evaluated according to the (undiscounted) average payoff; player $i^{\prime} s$ evaluation of the payoff stream $\pi_{i}\left(a_{t}\right), t=1,2, \ldots T$ is given by ${ }^{13}$

$$
\frac{1}{T} \sum_{t=1}^{T} \pi_{i}\left(a_{t}\right)
$$

Note that both models are examples of dynamic or Markovian games with the payoff-relevant state at time $t$ being the fixed actions and the next times of move of the non-movers. Let $\left(a_{t}, \theta_{t}\right)$ denote the state, $a_{t}$ being the previously determined actions and $\theta_{t}$ the next times of move. Obviously, in Model $1, \theta_{t}$ is exogeneously given while it is endogeneously determined in Model 2. Hence in Model 1 the state is always an element of $\Re^{2(N-1)}$ while in Model 2 it can range from the null vector (when every player is a mover) to an element of $\Re^{2 N}$ when no player is a mover.

For both models, strategies are defined in the usual way as historydependent action choices. A generic history up to period $t$, is a sequence $\left(a_{1}, \theta_{1}\right),\left(a_{2}, \theta_{2}\right), \ldots\left(a_{t}, \theta_{t}\right)$ where $\left(a_{1}, \theta_{1}\right)$ is the initial state. A $t-t h$ period strategy for a mover in that period is a history-dependent (mixed) action choice. A complete strategy is a strategy specification for every move period (and after every history). A strategy vector - one strategy for every player - defines in the usual way a (possibly probabilistic) action choice for the $t-t h$ period - and hence an expected payoff for each player in that period. In addition, in Model 2, it also defines the player assignment function - the timing of moves. The lifetime payoff for each player is then defined via the undiscounted average of these periodic expected payoffs (as given by Eq. 1).

Since the game has a finite horizon, Subgame Perfect Equilibrium (SPE) is determined via backward induction in both models. Consider period $T$ and a state $\left(a_{T}, \theta_{T}\right)$. Players who have a move in that period play a

\footnotetext{
${ }^{13}$ Everything that follows also applies to the case of discounting, under discount factor $\delta$. In that case the appropriate evaluation of lifetime payoffs is $\frac{1-\delta}{1-\delta^{T}} \sum_{t=1}^{T} \delta^{t-1} \pi_{i}\left(a_{t}\right)$.
} 
simultaneous move game taking as given the fixed actions of the non-movers. If only one player has a move - as would be the case in the exogenous move model - then she picks a best response action at that state.

The equilibria in the family of last period subgames then determine equilibria in subgames involving the two periods $T-1$ and $T$. And so on. Note that in both models, the set of SPE payoffs is always history-independent; the set of payoff vectors that can arise as a SPE in the subgame that starts at date $t$ and history $\left(a_{1}, \theta_{1}\right),\left(a_{2}, \theta_{2}\right), \ldots\left(a_{t}, \theta_{t}\right)$ only depends on the current state $a_{t}, \theta_{t}$ (and the number of remaining periods $\left.T-t\right) .{ }^{14} \mathrm{~A}$ specific equilibrium might depend on history and to allow for this dependence I will write a representative equilibrium payoff as $v_{i t}(. ; T)$ and an equilibrium strategy as $\alpha_{t}^{*}(. ; T)$, where the argument in both functions is history up to period $t$.

This paper will investigate properties the equilibrium choice and value sets must satisfy, when $T$ is "large".

\section{Alternating Moves}

This section contains the first main theorem of this paper - an $N$-player alternating move common interest game necessarily achieves efficiency within a uniformly bounded number of periods. That the theorem applies to any asynchronous game is shown in Section 5 .

Theorem 1 Consider a family of finite horizon alternating move common interest games that start from an initial state $a_{1}$ and last $T$ periods, $T=1,2$ ... There are numbers $\Gamma<\infty$ and (for every $\epsilon>0$ ) $\widehat{T}<\infty$ with the property that for any pure strategy SPE, any horizon and for any initial state

Action Convergence - at every period $t \geq \Gamma$, the Pareto-dominant action vector $\widehat{a}$ is played.

Value Convergence - each player's SPE payoff is within $\epsilon$ of the Pareto-dominant payoff for horizons longer than $\widehat{T}$, i.e., $v_{i}(. ; T)>\widehat{\pi}_{i}-$ $\epsilon$ for all $T \geq \widehat{T}$.

In other words, even if the players are "mis-coordinated" initially, such miscoordination will disappear within a fixed finite number of periods. Furthermore, the number of periods required to correct such miscoordination is independent of the initial state of the game, i.e., of what actions the players

\footnotetext{
${ }^{14}$ Note that in the perfect information setting of Model 1, optimal choices are, typically, Markovian and unique. The only way that a mixed strategy SPE - or a history-dependent $\mathrm{SPE}$ - can arise is from ties in the mover's payoffs.
} 
are initially committed to. It is also independent of the games's horizon; no matter whether the horizon is $T^{\prime}$ say, or $10 T^{\prime}$, within $\Gamma$ periods the Paretodominant vector starts getting played by all players. And, finally, $\Gamma$ is a uniform upper bound that applies to every SPE.

\subsection{Intuition for Theorem 1}

To develop intuition, I discuss two examples - a two-player game and then, to illustrate a problem that arises with more than two players, a threeplayer version of that game. First consider Example 2, from Section 2.1, reproduced here for easier access:

$\begin{array}{ccc}1 \backslash 2 & \widehat{a}_{2} & \widetilde{a}_{2} \\ \widehat{a}_{1} & 1,1 & -100,-1 \\ \widetilde{a}_{1} & -1,-100 & 0,0\end{array}$

Suppose it is player $1^{\prime} s$ turn to move, and she has inherited the action $\widehat{a}_{2}$. If the current period is the very last period in the game, she can do no better than play her half of the Pareto-dominant vector, i.e., play $\widehat{a}_{1}$. It is not too difficult to see by backward induction that $\widehat{a}_{1}$ is the best response at state $\widehat{a}_{2}$ - even if the current period is not the last one. Hence, and this is the first key idea, efficiency is an absorbing state - the best-response at state $\widehat{a}_{j}$ is to play $\widehat{a}_{i}$.

Suppose, on the other hand, that player 1 inherits the action $\widetilde{a}_{2}$. Her payoffs from playing $\widehat{a}_{1}$ are -100 followed by $1,1,1, \ldots$. From playing $\widetilde{a}_{1}$ her current payoff is 0 and her future payoff depends on how she and player 2 play in the resulting subgame (and that is known from backward induction). There are two relevant cases to consider: a) player 2 will play $\widehat{a}_{2}$ within the next 99 periods or b) he will not. In case a) it is better for player 1 to free-ride and let player 2 bear the loss since over the next 99 periods $0,0, \ldots 0,-1$ beats $-100,1, \ldots 1$. On the other hand, in case b), it is better for player 1 to initiate the switch to $\widehat{a}$ immediately since over the 100 or more periods $0,0, \ldots 0,-1$ is worse than $-100,1, \ldots 1$. The second key idea then is that player $i$ - who has the option to lock-in efficiency by playing $\widehat{a}_{i}$ today - will pass on that option only if he knows he can free-ride to efficiency within a bounded period. In particular, what is important is not how many periods are left in the game but how many periods are left before the current mover can free-ride. ${ }^{15}$

\footnotetext{
${ }^{15}$ In Example 2, the equilibrium is the following: neither player switches to $\widehat{a}_{i}$ when
} 
Consider now a three-player version of Example 2.

$$
\begin{array}{cccccc}
1 \backslash 2 & \widehat{a}_{2} & \widetilde{a}_{2} & 1 \backslash 2 & \widehat{a}_{2} & \widetilde{a}_{2} \\
\widehat{a}_{1} & 1,1,1 & -100,-1,-100 & \widehat{a}_{1} & -100,-100,-1 & -200,-2,-2 \\
\widetilde{a}_{1} & -1,-100,-100 & -2,-2,-200 & \widetilde{a}_{1} & -2,-200,-2 & 0,0,0 \\
\multicolumn{4}{c}{3 \text { plays } \widehat{a}_{3}} & & 3 \text { plays } \widetilde{a}_{3}
\end{array}
$$

As before, the Pareto-dominant action vector is $\widehat{a}$ whereas a Paretodominated Nash equilibrium is $\widetilde{a}$. The analog of the first step above is that once two of the players become coordinated, the three stay coordinated forever thereafter; for example, if ever the game reaches the state $\left(\widehat{a}_{2}, \widehat{a}_{3}\right)$ and it is player $1^{\prime} s$ turn to move -1 can do no better than play $\widehat{a}_{1}$.

But the problem is that it now takes two to tango, i.e., neither player 2 nor 3 can unilaterally create the state $\left(\widehat{a}_{2}, \widehat{a}_{3}\right)$. Indeed player 2 might not want to get the ball rolling (by playing $\widehat{a}_{2}$ ) if he anticipates that 3 would not play $\widehat{a}_{3}$ (and note that he gets the worst payoff of -200 by playing $\widehat{a}_{2}$ against $\left.\left(\widetilde{a}_{1}, \widetilde{a}_{3}\right)\right)$. In turn, player 3 might not play $\widehat{a}_{3}$ since that too is very costly it generates the second worst payoff of -100 - and 3 might prefer someone else bear that cost instead. Furthermore, since actions are reversible every two periods, even if a player has currently chosen the efficient action other players may not have assurance that such an action would not soon be reversed. And so it appears that for want of "coordinated sacrifices" the kingdom might be lost.

Suppose that nevertheless 2 plays $\widehat{a}_{2}$ and creates the state $\left(\widetilde{a}_{1}, \widehat{a}_{2}\right)$ for player 3. Note, player 3 could swallow the sacrifice of 100 and lock the system into the Pareto dominant vector $\widehat{a}$ thereafter. The logic of Example 2 tells us that player 3 will pass only if she anticipates that a state such as $\left(\widehat{a}_{1}, \widehat{a}_{2}\right)$ will arise in the next $\Gamma_{1}(\leq 99)$ periods. ${ }^{16}$

In turn we can now back up to player 2. Starting from the Paretodominated action vector $\widetilde{a}$, he can a) move the system to semi-efficiency - state $\left(\widetilde{a}_{1}, \widehat{a}_{2}\right)$ - in one step and he will know b) that within $\Gamma_{1}$ periods thereafter the system will move to full efficiency - state $\left(\widehat{a}_{i}, \widehat{a}_{j}\right)$. In other words, he can unilaterally move the system from zero efficiency to full efficiency in at most $\Gamma_{1}+1$ periods. Since, thankfully, it takes only one to

there are 100 or fewer periods remaining. The mover switches when there are 101, 103, $105, \ldots$ periods remaining but free-rides when there are $102,104,106, .$. periods remaining.

${ }^{16}$ By playing $\widehat{a}_{3}$, player 3 guarantees a payoff stream of $-100,1,1,1, \ldots$ By playing $\widetilde{a}_{3}$, player 3 gets at most a payoff stream of $-2,0,0,0, \ldots .$. till such time as the Paretodominant action gets played (and 1 thereafter). Evidently, player 3 will play $\widehat{a}_{3}$ unless she is sure that she can free-ride within 99 periods. 
tango, player 2 will pass on such a unilateral initiation only if he is sure that somebody else will initiate efficiency soon. ${ }^{17}$

\subsection{Proof of Theorem 1}

\section{Step 1 - Full Efficiency is An Absorbing State}

Lemma 2 In any SPE of any finite horizon alternating move common interest game $\langle T, G\rangle$, regardless of the number of periods left, the unique best response of every player $i$ upon inheriting the Pareto-dominant action vector $\widehat{a}_{j}, j \neq i$, is to play her part of the Pareto-dominant vector, i.e., for $i=1$, $2, \ldots N, i \neq j$, and for all $t, \alpha_{i t}^{*}\left(\widehat{a}_{j}, T\right)=\widehat{a}_{i}$.

Proof: The proof is by induction. Suppose that $t=T$. Evidently, the lemma holds. Suppose therefore that it holds for all $t=T, T-1, . . T-n+1$. Consider $t=T-n$. Suppose that player $i$ inherits the Pareto-dominant action $\widehat{a}_{j}$ as the fixed action of every other player. Her highest immediate payoff arises from playing $\widehat{a}_{i}$. By the induction hypothesis, her highest future payoff also arises from playing $\widehat{a}_{i}$ - since that action will then get continued for the remainder of the game. $\diamond$

\section{Step 2 - An Induction Argument for Efficiency Buildup}

The next step, that no matter where it starts, play must proceed to the absorbing state in which all future movers are committed to the efficient action will also be proved by induction but induction on the "efficiency level." Call that state in which $N-1$ players are committed to $\widehat{a}_{i}$ the " $N-1$ efficient" state. I will define a notion of intermediate efficiency and show that if the efficiency level is, say, $m$ then the current mover can always increase it to at least $m+1$. By the induction hypothesis he can therefore guarantee that full efficiency will be attained within, say, $\Gamma_{m+1}$ periods. Hence in any best-response his lifetime payoffs have to be at least

$$
\left(T-\Gamma_{m+1}-1\right) \times \widehat{\pi}_{i}+\left(\Gamma_{m+1}+1\right) \times \underline{\pi}_{i}
$$

\footnotetext{
${ }^{17}$ For instance, player 2 - by playing $\widehat{a}_{2}$ - can guarantee a lifetime payoff stream of $-200,-200,-200, \ldots 1,1,1, \ldots$ (where the sequence of -200 can last at most 100 periods - see the previous footnote). On the other hand, by playing $\widetilde{a}_{2}$ he can get at most a payoff stream of $0,0, \ldots$ till such time as the Pareto-dominant action vector gets locked in. Clearly, player 2 has to anticipate that he can free-ride within $200 \times 100$ periods else he is better off by initiating efficiency.
} 
where $\underline{\pi}_{i}$ is the lowest payoff in the game for player $i$. That immediately implies that there must be a bound, say $\Gamma_{m}$, such that full efficiency is attained within $\Gamma_{m}$ periods starting from an $m$-efficient state. Indeed along such a path the highest possible lifetime payoff for player $i$ is

$$
\left(T-\Gamma_{m}\right) \times \widehat{\pi}_{i}+\Gamma_{m} \times \bar{\pi}_{i}
$$

where $\bar{\pi}_{i}$ is the second-highest payoff in the game for player $i$. The $\Gamma_{m}$ bound is therefore determied by the inequality

$$
\left(T-\Gamma_{m}\right) \times \widehat{\pi}_{i}+\Gamma_{m} \times \bar{\pi}_{i} \geq\left(T-\Gamma_{m+1}-1\right) \times \widehat{\pi}_{i}+\left(\Gamma_{m+1}+1\right) \times \underline{\pi}_{i}
$$

First, a definition.

Definition $A$ state $a_{t}$ is said to be $N-1$ efficient if all $N-1$ actions that are fixed at date $t$ are fixed at $\widehat{a}_{i}$. If not all actions are fixed at $\widehat{a}_{i}$, then a state is said to be $(m, k)$ - order efficient when $i)$ exactly $m$ components are equal to $\widehat{a}_{i}, m=0,1, \ldots N-2$ and ii) the first period for which a fixed action is in fact not equal to $\widehat{a}_{i}$ is $t+k, k=1, \ldots N-1$.

In order to use induction on the grid $(m, k)$ we need a linear order and I will use the following lexicographic order:

Definition The highest efficiency number is $(N-1)$. Among two efficiency numbers $(m, k)$ and $\left(m^{\prime}, k^{\prime}\right)$ - of relevance when $\max \left(m, m^{\prime}\right)<N-1$ - $(m, k)$ is greater than $\left(m^{\prime}, k^{\prime}\right)$ iff either i) $m>m^{\prime}$ or ii) $m=m^{\prime}$ and $k<k^{\prime}$.

Lemma 3 For every $(m, k), m=0, \ldots N-2, k=1, \ldots N-1$ there is a number $\Gamma_{m, k}<\infty$ with the following property: for any pure strategy subgame perfect equilibrium and in any subgame that starts from an $(m, k)$ efficient state, full efficiency is achieved within at most $\Gamma_{m, k}$ periods, i.e., $\alpha_{t}^{*}=\widehat{a} \forall t$ $\geq \Gamma_{m, k}$.

Proof: The proof is by induction on the efficiency level. Note that the case where the state is $N-1$ order efficient is the content of the previous lemma. The next level down in terms of efficiency is $(N-2,1)$. Without loss of generality, suppose the current mover is player 1 . We therefore have the state $\left(\widetilde{a}_{2}, \widehat{a}_{3}, \ldots \widehat{a}_{N}\right)$ - player 2 who will move in the next period is the only one not committed to the efficient action. Evidently, player 1 can pick $\widehat{a}_{1}$ as her action thereby leaving player 2 with an $N-1$ efficient state which will be then maintained forever after. By doing so her payoff will be at least

$$
(T-1) \times \widehat{\pi}_{1}+\underline{\pi}_{1}
$$


where $\underline{\pi}_{1}$ is the lowest payoff for player 1 in the stage game. If player 1 does not choose the efficient action it must be because full efficiency will be attained in any case within $\Gamma_{N-2,2}$ periods - after she passes on the current opportunity - where that bound is defined by

$$
\left(T-\Gamma_{N-2,1}\right) \times \widehat{\pi}_{1}+\Gamma_{N-2,1} \times \bar{\pi}_{1} \geq(T-1) \times \widehat{\pi}_{1}+\underline{\pi}_{1}
$$

where $\bar{\pi}_{1}$ is the highest payoff for player 1 among action vectors other than the efficient one (whose payoff is $\widehat{\pi}_{1}$ ). Of course, the inequality can be simplified to

$$
\widehat{\pi}_{1}-\underline{\pi}_{1} \geq \Gamma_{N-2,1} \times\left(\widehat{\pi}_{1}-\bar{\pi}_{1}\right)
$$

Suppose now that the conclusion holds for all $(m, k)$ efficient states above a cut-off level $(\bar{m}, \bar{k})$,for some $\bar{m} \in(0, \ldots N-2)$. Consider now a state $\left(m^{\prime}, k^{\prime}\right)$ that is less efficient than that cut-off by one order. There are two cases:

Case $1-m^{\prime}=\bar{m}-1$ (which means $k^{\prime}=1$ ). The argument is very similar to that for the $(N-2,1)$ case that we just looked at.

The mover can turn the state into an $(\bar{m}, k)$ efficient state in the very next period - for some $k$ - by picking the efficient action on his move. By the induction hypothesis the payoffs to this action are at least as large as

$$
\left(T-\Gamma_{\bar{m}, k}-1\right) \times \widehat{\pi}_{1}+\left(\Gamma_{\bar{m}, k}+1\right) \times \underline{\pi}_{1}
$$

If the mover passes on this efficient action it must be because full efficiency will be attained in any case within $\Gamma_{m^{\prime}, k^{\prime}}$ periods where that bound is defined by

$$
\left(T-\Gamma_{m^{\prime}, k^{\prime}}\right) \times \widehat{\pi}_{1}+\Gamma_{m^{\prime}, k^{\prime}} \times \bar{\pi}_{1} \geq\left(T-\Gamma_{\bar{m}, k}-1\right) \times \widehat{\pi}_{1}+\left(\Gamma_{\bar{m}, k}+1\right) \times \underline{\pi}_{1}
$$

or

$$
\left(\Gamma_{\bar{m}, k}+1\right)\left(\widehat{\pi}_{1}-\underline{\pi}_{1}\right) \geq \Gamma_{m^{\prime}, k^{\prime}} \times\left(\widehat{\pi}_{1}-\bar{\pi}_{1}\right)
$$

Case $2-m^{\prime}=\bar{m}$ (which means $k^{\prime}=\bar{k}+1$ ). Again the mover can unilaterally increase efficiency (to $\left(m^{\prime}, \bar{k}\right)$ by playing the efficient action. The rest of the argument is identical to what we saw for Case 1.

Hence the lemma is proved and so is the theorem. $\diamond$

Remark 1: Lemma 1 - that Pareto-dominance is an absorbing state and that Pareto-dominant play starts the moment all players but one are playing $\widehat{a}_{i}$ - is a general result for all finite horizon asynchronous and asynchronisable games. This result is untrue when the horizon is infinite since in that setting 
we cannot use backward induction; it is always possible that after playing $\widehat{a}$ for a long time, player $i$ might switch away simply because he believes that player $j$ will do the same thing the next period. The more complicated step, especially when we consider asynchronisable games, is the second step in the proof above, the one that shows that this absorbing state must be reached.

Remark 2: A natural question that arises is: how large can the period of miscoordination $\Gamma$ be? A crude upper bound is suggested by the analysis

above; it must be that $\Gamma \leq \Delta+\Delta^{2}+\ldots \Delta^{M}$ where $\Delta=\frac{\bar{\pi}_{k}-\underline{\pi}_{k}}{\widehat{\pi}_{k}-\bar{\pi}_{k}}$ and $M$ is the cardinality of the set of efficiency levels.

Remark 3: The theorem above is for pure strategy SPE. A very similar result applies to mixed-strategy SPE. This result is stated and discussed in Section 5.

\section{Endogenous Asynchronisable Moves}

This section considers the second model of section 2, a model that allows players to choose their times of move. The main theorem shows that even in this case full efficiency is achieved within a uniformly bounded number of periods by every SPE. Hence, what is important for the selection result is not that players be forced to asynchronise but rather that they have the option to do so.

Theorem 4 Consider any family of $N$-player endogenous asynchronisable common interest games $\langle T, G\rangle$ with initial state $\left(a_{0}, \theta_{0}\right)$ and horizon $T=$ $1,2 \ldots$ There are numbers $\Gamma<\infty$ and (for every $\epsilon>0$ ) $\widehat{T}<\infty$ with the property that for any pure strategy SPE, any horizon, and for any initial state

Action Convergence - the Pareto-dominant action vector $\widehat{a}$ must be played in all but at most $\Gamma$ periods.

Value Convergence - each player's SPE payoff is within $\epsilon$ of the Pareto-dominant payoff for horizons longer than $\widehat{T}$, i.e., $v_{i}(. ; T)>\widehat{\pi}_{i}-$ $\epsilon$ for all $T \geq \widehat{T}$.

The ideas behind the general result for asynchronisable games are quite similar to that for alternating move games. Again the argument is in two steps. There is an easy step to show that if the players ever get to an alternating move timing structure - a single mover in the current period and one each in the next $N-1$ periods - and each of the future movers is committed to the Pareto-dominant action, then the unique best response 
for the current mover is to play her Pareto-dominant action as well and commit to moving next in $N$ periods. In other words, alternating moves plus efficiency is an absorbing state. The second and more difficult part is to show that this absorbing state must be reached in finite time.

I will argue the second step by induction on the level of efficiency. The exact definition of efficiency level is a bit more complex in this model relative to alternating moves - and so I shall explain that with some care in the next subsection.

\subsection{An Efficiency Index}

Recall that $\Theta$ is the set of dates that are available at date $t$. By extension let $\Theta^{c}$ be its complement, i.e., the set of dates already committed to (the subset of $t+1, t+2 \ldots t+N-1$ such that at every one of those periods there is at least one mover). In turn, let $\widehat{\Theta}$ be the subset of $\Theta^{c}$ that has at least one mover committed to $\widehat{a}_{i}$.

To fix ideas, consider the following two scenarios:

Example 1 - There are four players in a game, and players 3 and 4 are committed to moving next in period $t+3$. Players 1 and 2 have a move in the current period $t$. In this case, $\Theta^{c}=\{t+3\}$. If either - or both - of players 3 and 4 are committed to $\widehat{a}_{i}$, then $\widehat{\Theta}=\Theta^{c}$.

Example 2 - In the same example if players 3 and 4 are both committed to some action other than the Pareto-dominant one, then, again $\Theta^{c}=\{t+3\}$ but $\widehat{\Theta}=\emptyset$.

Let me now turn to the efficiency index. Recall from Section 3 that this index looks at two things - a) how many of the future periods have a player committed to the efficient action and b) what is the first future period at which an inefficient action will expire. In other words, a) counts the cardinality of $\widehat{\Theta}$ while b) notes the smallest time-period in the set $\Theta^{c} / \widehat{\Theta}$, the set of inefficient commitments.

Definition Suppose there is a mover at date $t$. A state $\left(a_{t}, \theta_{t}\right)$ is said to be $N-1$ efficient if the cardinality of $\widehat{\Theta}$ is $N-1$. Otherwise, a state is said to be $(m, k)$ - order efficient if $i)$ the cardinality of $\widehat{\Theta}$ is $m, m=0,1$, ... $N-2$ and ii) the first period for which there is no fixed action equal to $\widehat{a}_{i}$, i.e., the first period in the set $\Theta^{c} / \widehat{\Theta}$ is $t+k, k=1, \ldots N-1$. When the set $\Theta^{c} / \widehat{\Theta}$ is empty, we define $k=N$.

Remark: Hence, for the timing structure of Example 1, $m=1, k=4$ while in that of Example 2, $m=0, k=3$.

There is one other possibility - there may not be any mover in the current 
period $t$. In that case I will make the natural reduction and define the efficiency of the state as the efficiency of the first period thereafter in which there will be movers.

Definition Suppose there is no mover at date $t$. Suppose further that $t+s$ is the smallest element of $\Theta^{c} .^{18}$ The efficiency of state $\left(a_{t}, \theta_{t}\right)$ is then defined to be the efficiency of the resultant state at date $t+s$.

The ordering on $(m, k)$ will be identical to that discussed in Section 3. The highest efficiency number will be $(N-1)$. Among two efficiency numbers $(m, k)$ and $\left(m^{\prime}, k^{\prime}\right)$ - of relevance when $\max \left(m, m^{\prime}\right)<N-1$ $(m, k)$ is greater than $\left(m^{\prime}, k^{\prime}\right)$ iff either i) $m>m^{\prime}$ or ii) $m=m^{\prime}$ and $k<k^{\prime}$.

We are now ready to prove the theorem.

\subsection{Proof of the Theorem}

Proof: The first step is an analog of Lemma 2.

Lemma 5 Suppose that the current state is $N-1$ - efficient. In any SPE continuation of $\langle T, G\rangle$, regardless of the number of periods left, the Paretodominant action vector must get played in every period thereafter.

Proof: The proof is identical to that for Lemma 2. Suppose that $t=T$. Evidently, the lemma holds. Suppose therefore that it holds for all $t=T$, $T-1, . . T-k+1$. Consider $t=T-k$. Suppose that player $i$ inherits an $N-1$ efficient state. Her highest immediate payoff arises from playing $\widehat{a}_{i}$. Furthermore, if she commits to $\widehat{a}_{i}$ for the next $N$ periods, she hands over an $N-1$ efficient state to the single mover next period. By the induction hypothesis, play will then be of the Pareto-dominant action vector $\widehat{a}$ for the remaining periods, i.e., the highest future payoff will be realized. $\diamond$

The next lemma establishes that the efficient action vector must be played in all but a set of uniformly bounded periods:

Lemma 6 For every $(m, k): m=0, \ldots N-2, k=1, \ldots N$ there is a number $\Gamma_{m, k}<\infty$ with the following property: for any pure strategy subgame perfect equilibrium and in any subgame that starts from an $(m, k)$ efficient state, full efficiency is achieved within at most $\Gamma_{m, k}$ periods.

Proof: Suppose, to begin with, that there is at least one mover in the current period. The proof is by induction on the efficiency level. Note that

\footnotetext{
${ }^{18}$ In this case by definition $\Theta$ is non-empty.
} 
the case where the state is $N-1$ efficient is the content of the previous lemma. The next level down in terms of efficiency is $(N-2,1)$. Without loss of generality, suppose the current mover is player 1 . We know that there must be exactly one mover in every period, $t, t+1, \ldots t+N-1$, the $t+1$ mover must be committed to an inefficient action and every one of the others must be committed to the efficient action. ${ }^{19}$

Player 1 has the option of commiting to move again in period $t+N$ and choosing $\widehat{a}_{1}$ as his fixed action. This increases the efficiency level to $N-1$. By the previous lemma, that will be then maintained forever after. By an argument identical to that in Lemma 3 it follows that there must be a bound $\Gamma_{N-2,1}$ within which full efficiency must be achieved and it is defined by

$$
\widehat{\pi}_{1}-\underline{\pi}_{1} \geq \Gamma_{N-2,1} \times\left(\widehat{\pi}_{1}-\bar{\pi}_{1}\right)
$$

(where $\widehat{\pi}_{1}, \underline{\pi}_{1}$, and $\bar{\pi}_{1}$ are, recall, the highest, lowest and second-highest payoff for player 1 in the stage game).

Suppose now that the lemma holds for efficiency levels above a cut-off, say $(\bar{m}, \bar{k})$, i.e., that it holds for all states $(m, k)$ such that either i) $m>\bar{m}$ or ii) $m=\bar{m}$ and $k \leq \bar{k}$. Consider now a state $(m, k)$ that is less efficient than $(\bar{m}, \bar{k})$ by one order. Again, without loss, assume that one of the current movers is player 1 . There are three cases:

Case $1-m=\bar{m}-1$ (which means $k=1$ ). Player 1 can then turn the state into an $(\bar{m}, k)$ efficient state in the very next period - for some $k$ - by picking the efficient action on his move and committing to move again in period $t+N .^{20}$ By the induction hypothesis the payoffs to this action are at least as large as

$$
\left(T-\Gamma_{\bar{m}, k}-1\right) \times \widehat{\pi}_{1}+\left(\Gamma_{\bar{m}, k}+1\right) \times \underline{\pi}_{1}
$$

If player 1 passes on this efficient action it must be because full efficiency will be attained in any case within $\Gamma_{m, k}$ periods where that bound is defined by

$$
\left(T-\Gamma_{m, k}\right) \times \widehat{\pi}_{1}+\Gamma_{m, k} \times \bar{\pi}_{1} \geq\left(T-\Gamma_{\bar{m}, k}-1\right) \times \widehat{\pi}_{1}+\left(\Gamma_{\bar{m}, k}+1\right) \times \underline{\pi}_{1}
$$

\footnotetext{
${ }^{19}$ This follows from the following facts: i) by assumption, there is at least one mover in the current period, ii) since $k=1$, there is at least one mover in the next period, and iii) since $m=N-2$, there are an additional $N-2$ players committed to the efficient action and committed to distinct periods. Since in all there are $N$ players, it follows that there is one each in the periods $t$ through $t+N-1$.

${ }^{20}$ If there are multiple movers in period $t$ and some of those movers also pick the efficient action and a period different from $t+N$, then in fact the state can become more efficient than $(\bar{m}, k)$. The same argument applies however.
} 
Case $2-m=\bar{m}, 1<k<N$ (and, of course, $k=\bar{k}+1$ ). Player 1 can pick the efficient action and commit to move again in period $t+N$. If the next date after $t$ at which there are committed movers is an element of $\widehat{\Theta}$, i.e., if the next move date has an efficient commitment, then such a choice by player 1 increases efficiency to $(\bar{m}, \bar{k}) \cdot{ }^{21}$ On the other hand, if the next date at which there are committed movers is not an element of $\widehat{\Theta}$, i.e., if the next move date has no efficient commitments, then such a choice by player 1 increases efficiency to at least $(\bar{m}+1, \bar{k})$. The rest of the argument is identical to what we saw for Case 1.

So far the argument has been essentially identical to the alternating move case. We now deal with one new case that arises for asynchronisable games: even at an intermediate efficiency level, there may not be any players committed to inefficient actions:

Case $3-m=\bar{m}, k=N$ In this case the sets of committed dates and the set of committed dates with efficient commitments are identical, i.e., $\Theta^{c}=\widehat{\Theta}$ and both sets might, of course, be empty.

There are three sub-cases that I need to consider. In each one the same action - pick $\widehat{a}_{1}$ and commit to it till $t+N$ - increases the efficiency level. Call that action $\left(\widehat{a}_{1}, N\right)$.

Case $3 \mathrm{a}$ - If the sets are empty, i.e., if all players have a move in the current period, $m=0$. Evidently $\left(\widehat{a}_{1}, N\right)$ makes $m>0$.

Case $3 \mathrm{~b}$ - Suppose instead that the sets are non-empty and consider first the case where there are multiple movers in the current period - one of them being, say, player 1. If all of the other movers pick an inefficient action then $k$ becomes less than it is currently $(=N)$, i.e., the efficiency index strictly increases. If at least one of the other current movers picks the efficient action as well then Player 1 can always pick a date that is distinct. ${ }^{22}$ Consequently the index increases.

Case $3 \mathrm{c}$ - If, on the other hand, there is a single mover in the current period then $\left(\widehat{a}_{1}, N\right)$ maintains efficiency. Within at most $N-1$ periods, there has to be multiple movers. Then we are in case $3 \mathrm{~b}$.

So in all cases a mover can immediately - or within $N-1$ periods - strictly increase the efficiency index. By the induction hypothesis she can therefore implement full efficiency within at most $\Gamma_{\bar{m}, \bar{k}}$ periods. The argument of

\footnotetext{
${ }^{21}$ Again efficiency may actually increase further than that if there are multiple movers - other than player 1 - at date $t$ and some of those other movers asynchronize and commit to the efficient action themselves.

${ }^{22}$ Note that if there are $l$ players who are movers in the current period then there are at least $N-l$ open time-periods to which commitments can be made.
} 
Case 1 then says that she will pass on doing so only if she believes that she can free-ride within a uniformly bounded number of periods anyway.

Suppose, finally, that there is no mover in the current period. By definition, within $N-1$ periods, there will be. The above argument can then be applied.

Hence the lemma is proved and so is the theorem. $\diamond$

\section{Three Other Results}

This section presents two results that apply to the exogenous asynchronous move model and one to the endogenous asynchronisable model. To begin with I show that the first main result, Theorem 1, holds for general exogenous asynchronous games (Theorem 1 showed that it holds for alternating move games). Within that same model I then show that the result also holds for mixed strategy equilibria (Theorem 1 showed that it holds for pure strategy SPE).

Thereafter I turn to the endogenous asynchronizable model. I discuss a slighly more general version of that model than the one that was discussed in Section 4 and and show that the main theorem - Theorem 4 - continues to apply in that more general model.

\subsection{Exogenous Asynchronous Games}

To recall, in this model there is a player assignment function $X:\{1, \ldots T\} \rightarrow$ $\{1, \ldots N\}$ such that $X(t)$ is the sole mover at date $t$. From that one can deduce individual player assignment functions $\Phi_{i}$, one for each player, that specify for every period $t$ and every player $i$ a next time of move for that player, $\Phi_{i}(t)$. Since I am interested in arbitrary but long horizons, the domain of these functions will be the set of all positive integers. If the game under study is $T$ periods long, then (at most) the first $T$ components of $\Phi_{i}$ are used.

The main result of this sub-section shows that as long as the move functions satisfy a mild condition that I call unbiasedness, then finite horizon play picks out the Pareto-dominant equilibrium. To set the stage for the definition, let $\lambda_{i}$ denote the maximum waiting time for player $i$ :

$$
\lambda_{i}=\sup _{t}\left[\Phi_{i}(t)-t\right]
$$

Definition The functions $\Phi_{i}$ are unbiased if for every player $i, \lambda_{i}$ is finite. Denote the largest $\lambda_{i}$ value, $M(<\infty)$. 
Evidently an alternating move assignment function is unbiased - in that case $M=N$. An example of a biased assignment function is an alternating and increasing function - one in which the $N$ players alternate their moves in the first "round"; then each player gets to move twice in succession (and play proceeds by turn through player numbers 1 to 2 to 3 and so on). Then in the third "round" the procedure is repeated except that each player gets to move thrice in succession. By round $K$ each player moves $K$ times in succession.

Unbiasedness is a condition that must necessarily be satisfied by the assignment function in order for the convergence to efficiency result to hold. To see this, note that if in the current round of play player $i$ has $K$ successive moves then, in any history-independent SPE, in the first $K-1$ of those periods he is going to play a myopic best response to the fixed actions of the other players. After all they do not get to change their moves and any action that he takes in these first $K-1$ periods has no effect on subsequent play. Since I consider all equilibria, including history-independent ones, I will therefore not be able to prove convergence to efficiency for all SPE with such a move function. Hence, unbiasedness is a necessary condition for efficient selection.

It turns out to be a sufficient condition as well:

Theorem 7 Consider any family of $N$-player finite horizon asynchronous common interest games $\langle T, \Phi, G\rangle$ that start from an initial state $a_{0}$ and last $T$ periods, $T=1,2 \ldots$ and whose move functions are unbiased. There are numbers $\Gamma<\infty$ and (for every $\epsilon>0$ ) $\widehat{T}<\infty$ with the property that for any pure strategy SPE, any horizon and for any initial state, action and value convergence holds (in the sense of Theorem 1).

The proof of the above theorem mirrors the proof of the analogous result for alternating move games. ${ }^{23}$ Again the key ideas are two: a) that $\widehat{a}$ is an absorbing state, and b) that players can build towards the Pareto-dominant vector gradually, going from zero efficiency to partial and then full efficiency. Part b) is a little more delicate than in the alternating move case. After all, player $k$ may have just moved and yet may have a next move before a number of other players - a possibility that is ruled out under alternating moves. In turn, this phenomenon can create a potential problem because player $k$ cannot increase the efficiency of the system by switching to $\widehat{a}_{k}$ in

\footnotetext{
${ }^{23}$ And on account of the similarity between the two proofs, I consign the proof to the dungeon of section 7 .
} 
the current period. Unbiasedness plays a key role in proving this second step.

\subsection{Mixed Strategy SPE}

I now prove a result for mixed strategy SPE in the exogenous asynchronous model. Clearly the result also covers mixed strategy SPE in alternating move games.

In analyzing pure strategy SPE we found the following tension in a mover's incentives: she would rather free-ride and would be willing to wait on the Pareto-dominant payoffs just so as to not bear the costs of switching. On the other hand, she would rather switch now if she could not free-ride relatively soon. A similar trade-off shows up in mixed strategy SPE; for a high enough probability of free-riding in the immediate future, a player prefers to wait while for a low probability she prefers to switch. In equilibrium, she is indifferent and herself switches with a probability that makes the other players then indifferent between switching and free-riding. Consequently, the system can take a while to transit to the Pareto-dominant state. However, long waits are low probability events.

Theorem 8 Consider any family of $N$-player finite horizon asynchronous common interest games $\langle T, \Phi, G\rangle$ that start from an initial state $a_{0}$ and last $T$ periods, $T=1,2 \ldots$ and whose move functions are unbiased.

Action Convergence: For every $\theta>0$ there is a number $\Gamma(\theta)<\infty$ such that in any SPE, Prob $\left\{a_{t}=\widehat{a}, \forall t \geq \Gamma(\theta)\right\} \geq 1-\theta$.

Value convergence: There is a number $\widehat{T}<\infty$ such that in any SPE - and for any initial state - each player's payoff is within $\epsilon$ of the Paretodominant payoff for horizons longer than $\widehat{T}$.

Since the logic of the proof is merely a probabilistic version of the proof of Theorem 1, I relegate it to Section 7.

The theorem admits the possibility that $\Gamma(\theta)$ might drift up to $\infty$ as $\theta$ $\downarrow 0$, i.e., that is in some games with some mixed strategy equilibria, players might stay uncoordinated for a long period of time - albeit with a very small probability. It is however the best result that one can hope to get. An alternating move game analysis of Example 2 - reproduced yet again for easy reference - shows us why.

$$
\begin{array}{ccc}
1 \backslash 2 & \widehat{a}_{2} & \widetilde{a}_{2} \\
\widehat{a}_{1} & 1,1 & -100,-1 \\
\widetilde{a}_{1} & -1,-100 & 0,0
\end{array}
$$


Consider starting with the inefficient action vector $\widetilde{a}$. If $T=1$, clearly a best response for the mover is to continue to play $\widetilde{a}_{k}$. Even if $T=2$, it is still better to get a total payoff of 0 by continuing to play $\widetilde{a}_{k}$, rather than to switch to $\widehat{a}_{k}$ and its associated total payoff of -99 . Indeed it is easy to see by backward induction that the unique best response to $\widetilde{a}$ is to play $\widetilde{a}_{k}$ as long as $T<101$. At $T=101$, the mover is indifferent between her two actions. Suppose that she picks $\widetilde{a}_{k}$ with probability $q$.

In that case, for the other player, at $T=102$, immediate switching yields a total of $-100+101=1$ while staying with $\widetilde{a}_{k}$ yields $0+q .0+(1-q)[-1$ $+100]=99(1-q)$. Evidently, the mover is indifferent at $T=102$ if the switch probability next period is $q=\frac{98}{99}$. Suppose that consequently the mover at date $T=102$ stays with $\widetilde{a}_{k}$ with probability $p$.

Now consider $T=103$. Immediate switching yields a total of $-100+$ $102=2$ while staying with $\widetilde{a}_{k}$ yields $0+p[0-100+100]+(1-p)[-1+$ 101]. The term in the second bracket exploits the fact that if the opponent does not switch in the next period then the current mover is indifferent between switching and not in the subsequent period after that; note that switching after two periods means there are only 100 periods remaining at that point and hence the total payoff is $-100+100$. The second total is hence $100(1-p)$. Evidently, the mover is indifferent at $T=102$ if the no-switch probability next period $p$ is $\frac{49}{50}$.

Indeed the case of $T=103$ is more generally true whenever $T>103$. Suppose for instance $T=104$. The way we have constructed the equilibrium, when there are 102 periods left the mover - the same one as at date 104 - is indifferent between switching and not switching. If she does in fact switch then, we are reduced to the following comparison: continue with $\widetilde{a}_{k}$ and with probability $p$ this yields 0 for the next two periods and -100 the period thereafter while with probability $1-p$ it yields $0,-1$ and 1 over the next three periods. Immediate switching yields $-100,1$, and 1 over the same three periods. Beyond the three periods, the continuations are identical, viz., 1 every period thereafter. Evidently, for $p=\frac{49}{50}$, a player is indifferent between her two actions.

These arguments yield the following proposition:

Proposition 9 In an alternating move version of Example 2, for any horizon length $T>103$ the following strategies constitute a mixed strategy SPE: 
$\alpha_{k t}^{*}\left(\widehat{a}_{j}\right)=\widehat{a}_{k}$ for all $t$ and

$$
\begin{aligned}
& \alpha_{k t}^{*}\left(\widetilde{a}_{j}\right)=\widetilde{a}_{k} w / \text { probability } \frac{49}{50}, t \leq T-103 \\
& \quad=\widetilde{a}_{k} w / \text { probability } \frac{98}{99}, t=T-102 \\
& \quad=\widetilde{a}_{k} w / \text { probability } 1, t>T-102
\end{aligned}
$$

Of course an implication of the proposition is that no matter how long a horizon we consider, there is always an equilibrium that having started at the inefficient action vector stays there with positive probability. On the good news side, this probability is small. Hence, as Theorem 8 tells us the equilibrium value is virtually that of the Pareto-dominant value.

\subsection{A More General Model 2}

In this subsection I generalize Model 2, the endogenous model. In particular, I generalize the model to allow for temporary asynchronizations. Recall in Model 2 that if a single mover - say player $j$ - in the current period chose a period $t+\zeta$ as her next time of move, then no player between periods $t+1$ and $t+\zeta-1$ is allowed to choose that period. This has the immediate consequence that $j$ moves alone not only at period $t+\zeta$ but forever after. Asynchronization cannot be temporary.

Moreover one can logically make the argument that in the scenario considered above, periods $t+1$ through $t+\zeta-2$ are different from period $t+\zeta-1$ in the following sense. Whereas at any one of the periods $t+1$ through $t+\zeta-2$ having a next move at $t+\zeta$ has a commitment aspect to it - after all the fixed action will remain fixed for at least one more period - that is not true at period $t+\zeta-1$. Once that penultimate period is arrived at there is no more commitment left in the action since the very next period it can be changed. This motivates the following idea:

Definition: A time $t$ strategy for any mover, say player $i$, is given by a pair $\left(a_{i t}, \theta_{i t}\right)$ where $a_{i t}$ is an element from the set $A_{i}$ and $\theta_{i t}$, the date of next move, is either an element of $\Theta_{t}$ or is the date $t+1^{24}$

Remark 1: The greater generality relative to Model 2 is that any period $t$ mover can choose to move again at date $t+1$ even if there was a prior commitment to that date by some other player. Of course a period $t$ mover can alternatively commit to a date beyond $t+1$ and those dates can only be chosen if no prior mover is committed to them.

\footnotetext{
${ }^{24}$ Recall the definition of $\Theta_{t}$. A date $t+\zeta$ is said to be available at $t$ if no player chose it as a time of next move in any of the periods $t-1, t-2, \ldots t-N+1$. Let $\Theta_{t}$ be the set of dates available at $t$.
} 
Remark 2: In this model, a player who asynchronizes by uniquely choosing $t+\zeta$ is not forever asynchronized. At date $t+\zeta-1$ movers can choose to move simultaneously with her in date $t+\zeta$. Likewise, she can choose to move simultaneously with the movers in $t+\zeta+1$. Hence, an alternating move structure is not an absorbing state. To see that, consider:

Example Alternating Moves Transiting to Simultaneous Moves: Initially, player 1 has a move at $t$, player 2 at $t+1$, player 3 at $t+2$ and so on. At his move time, player 1 can either make no commitment and move at $t+1$ or he can make the only possible commitment which is to move next at $t+N$. Suppose player 1 chooses $t+1$. And in the next period, he chooses $t+2$; and every period thereafter he chooses to move again in the very next period. The first time player 2 moves - at date $t+1$ - she also makes no commitment and moves again at $t+2$. And so on - within $N$ periods all the players transit to moving every period.

In this model the same result that has been proved for endogenous asynchronizable games holds:

Theorem 10 Consider the more general version of Model 2. There are numbers $\Gamma<\infty$ and (for every $\epsilon>0$ ) $\widehat{T}<\infty$ with the property that for any pure strategy SPE, any horizon, and for any initial state action and value convergence holds.

\section{This and That}

This section reviews the relevant literature, offers some policy implications and concludes.

\subsection{Literature}

There is a vast literature on the coordination problem both from a theoretical perspective as well as in terms of applications. The latter in particular is nicely summarized in Cooper (1999) and I can do no better than point the interested reader to that source especially if they are looking for macroeconomic applications. Coordination games have also been used in Industrial Organization especially to study markets with network externalities; a very good source on that issue is Tirole (1989).

There have also been numerous experiments run with coordination games. In a repeated game setting, the best known of these experiments were run 
by Van Huyck et. al. (1990). ${ }^{25}$ The authors repeatedly run a partnership/public provision game similar to the one in Example 4; the game has many Pareto-ranked Nash equilibria. They find that players do not play the Pareto-dominant equilibrium especially in late stages (although they do seem to in early periods). They also find that increasing the number of players makes the coordination problem worse. The finding that the Pareto-dominant equilibrium is not played by subjects is a conclusion shared by many experimental studies. Indeed based principally on the fact that ".. coordination failure can arise in experimental games .." (p. 19) Cooper concludes that coordination is indeed a robust and wide-spread problem. The results in this paper suggest a natural experiment using asynchronous coordination games. To my knowledge, no one has done such an experiment.

Turning to theoretical analyses, Lagunoff-Matsui (1997) show that in an infinite horizon alternating move pure coordination game, the Paretodominant outcome will get uniquely selected in an SPE provided the discount factor is high enough. Their result is very interesting but works off the key insight that in a pure coordination game computing an equilibrium is equivalent to solving a decision problem. This is because $\pi_{i}=\pi$ and hence the life-time discounted payoffs to a time-profile $a_{0}, a_{1}, \ldots a_{t}, .$. is the same for every player and is simply $\sum_{t=0}^{\infty} \delta^{t} \pi\left(a_{t}\right)$. Indeed the reult is untrue for common interest games (where $\pi_{i} \neq \pi$ ), i.e., in discounted infinite horizon alternating move common interest games, the Pareto-dominant action is not the unique equilibrium outcome. Alternating move games are examples of stochastic games - with the fixed actions being the state variable and common interest games satisfy the full dimensionality requirement of Dutta's (1995) folk theorem for stochastic games. Note that pure coordination payoffs (as a subset of the 45 degree line) do not satisfy the full dimensionality requirement but common interest payoffs do.

Lagunoff-Matsui (2002) have recently shown that their result can be extended in the following sense. Fix a pure coordination game. For a fixed but high discount factor (such that Lagunoff-Matsui (1997) applies), and a specified $\epsilon>0$, there is a neighborhood of games around the pure coordination game such that all equilibria of each such game is within $\epsilon$ of the Pareto-dominant payoff. This result works off the upper hemi-continuity of the SPE correspondence when one varies the stage-game payoffs.

The second important paper related to the current one is Gale (1995).

\footnotetext{
${ }^{25}$ Static coordination game experiments have been run by many authors; a nice study is Cooper et. al. (1990).
} 
Gale studies a model in which each player decides on a time at which to invest in a (public) project. The investment decision is a one-time decision and is irreversible, i.e., an investment once made cannot be withdrawn. There is a fixed cost to making an investment and there is a coordination element because the benefits to the project are increasing in the number of players who have already made the investment. Since there is discounting, the Pareto-dominant outcome is for all players to invest in period zero. However there are private incentives to delay the cost and get in on the project only after a sufficient number of other players have done so. The paper shows that although equilibria will in general have delay, as the length between contiguous periods goes to zero, all equilibria must converge to efficiency. ${ }^{26}$

What is very interesting about the paper is that the result was proved in an induction manner very similar to that in the current paper. Gale defines an efficiency index that counts the number of players who have already invested. Beyond a certain cut-off on that index it is a dominant strategy for all remaining players to invest. And then immediately short of that cut-off players do backward induction on the efficiency level, etc.

One important difference between the two papers however has to do with irreversibility. As Gale $(1995,2000)$ point out the result works critically off the fcat that investment cannot be withdrawn. Hence efficiency can only rise. By contrast in this paper no player is committed forever to any action. Indeed efficiency may well fall along certain equilibrium paths. ${ }^{27}$

Turning to other authors, Crawford (1995) analyzes the performance of boundedly rational adaptive decision rules in which agents play in period $t$ a simple average of their own play and a summary of the others' play in period $t-1$. He shows that such rules explain well the Van Huyck et. al. experimental conclusions but that risk dominance motivations do not. Aumann and Sorin (1989), Matsui (1991), and Anderlini (1991) undertake a very different objective; they try to find conditions (much as I do in the current paper) under which the Pareto-dominant action will get played as the unique equilibrium outcome. They do their exercises within the simultaneous move framework which explains in part why quite elaborate sets of assumptions underlie each result. ${ }^{28}$ In contrast to all these papers I consider

\footnotetext{
${ }^{26}$ An earlier and nice paper on joint provision of publice goods is Admati-Perry (1991).

${ }^{27}$ In Gale (2000), the analysis is extended to monotone games with positive spillovers, i.e., games in which actions can only increase (= irreversibility) and the payoff to any one player is greater if the others take higher actions. It is shown that not all payoffs are achievable in equilibrium but a subset called "approachable" are.

${ }^{28}$ For example, Aumann-Sorin prove their result only for two players, they require per-
} 
fully rational players (playing SPE).

\subsection{Policy Implications}

The first - and most immediate - implication of my results is that asynchronous choice makes a big difference. Hence, policy should allow asynchronicity (as in my endogenous model) or should enforce it (as in my exogenous model). To the extent that literal simultaneity of moves is a physical impossibility anyway this is not a demanding requirement. Of course, effective simultaneity can also arise from lack of information about others' (already selected) choices. Hence, my results can be seen as a call for "market transparency". Whenever there is common interest present, policy is best off making choices publicly and rapidly available to everyone. Asynchronicity allows a player to make a commitment and in that sense is more demanding than simultaneous setting. However my results suggest that policy need only allow short-period commitments (no bigger than the number of players).

A second broad implication of my results is that the finiteness of horizon is crucial and hence given a choice, say, between rolling policies and ones with a definite time-line, the latter should be preferred by policy-makers (whenever there is common interest). Finally, I showed that just one degree of common interest - an agreement on the best outcome and only that - is enough to uniquely select this social optimum. In particular, disagreements of all kinds and of varied intensities further down the rank-list are irrelevant to equilibrium selection. This suggests that designing an incentive scheme may not be too difficult a policy problem in some contexts.

This paper showed when the horizon is finite and play is (potentially) asynchronous, coordination is not a problem.

\section{Three Proofs}

\subsection{Proof of Asynchronous Theorem}

The proof is almost identical to that for Theorem 1, the alternating move case. I shall first define an appropriate index of efficiency for this model the index is very similar to that for alternating moves.

turbration of every strategy with a strategy of uniformy bounded recall and require that there be a positive probability that a myopic strategy gets played. Finally they only prove a result for Nash equilibrium. 
Definition $A$ state $a_{t}$ is said to be $N-1$ efficient if all $N-1$ actions that are fixed at date $t$ are fixed at $\widehat{a}_{i}$. If not all actions are fixed at $\widehat{a}_{i}$, then a state is said to be $(m, k)$ - order efficient when $i)$ exactly $m$ components are equal to $\widehat{a}_{i}, m=0,1, \ldots N-2$ and ii) the first period for which a fixed action is in fact not equal to $\widehat{a}_{i}$ is $t+k, k=1, \ldots M$.

Note that the only difference in the definition is that the first time that an inefficient action expires may be any time between $t+1$ and $t+M$ (rather than $t+1$ and $t+N-1$ as was the case with alternating moves). A direct implication of unbiasedness is that the upper bound $t+M$ is finite. On the grid $(m, k)$ we will use the same lexicographic order as before. ${ }^{29}$

The first thing to note is that for any set of move functions - biased or unbiased - Lemma 1 holds: if every other player last played $\widehat{a}_{i}$ and it is player $j^{\prime} s$ turn to move, irrespective of the number of periods left in the game, $j^{\prime} s$ unique best response is to play $\widehat{a}_{j}$. The second step - that this $N-1$ efficiency must be attained in finite time - is almost identical to the argument already presented in Lemma 2. Hence it is presented now in an abbreviated form.

Lemma 11 Consider any family of finite horizon asynchronous common interest games $\langle T, \Phi, G\rangle$ with unbiased assignment functions $\Phi_{i}$. For every $(m, k), m=0, \ldots N-1, k=1, \ldots$ there is a number $\Gamma_{m, k}<\infty$ with the following property: for any $T \geq \Gamma_{m, k}$, any pure strategy SPE and in any subgame that starts from an $m, k$ - order efficient state, full efficiency is achieved within at most $\Gamma_{m, k}$ periods.

Proof: As before, the proof is by induction on the efficiency level. $(N-1)$ efficiency is absorbing. So consider a lower efficiency level. There are two cases.

Case 1 - The $t$ period mover is not the mover at period $t+1$ : A mover can then play $\widehat{a}_{i}$ till his next time of move. That will increase the efficiency level. Then the analysis of Lemma 2 applies without change.

Case 2 - The $t$ period mover is also the mover at period $t+1$ : By the unbiasedness assumption, within $M$ periods it must be true that we arrive at Case 1. Hence the bound derived in Case 1 plus $M$ applies in this case.

We have the lemma and hence the asynchronous theorem. $\diamond$

\footnotetext{
${ }^{29}$ Among two efficiency numbers $(m, k)$ and $\left(m^{\prime}, k^{\prime}\right)$ - of relevance when $\max \left(m, m^{\prime}\right)<$ $N-1-(m, k)$ is greater than $\left(m^{\prime}, k^{\prime}\right)$ iff either i) $m>m^{\prime}$ or ii) $m=m^{\prime}$ and $k<k^{\prime}$.
} 


\subsection{Proof of Mixed Strategy Theorem}

The theorem clearly works if we start with an $N-1$ efficient state. Suppose then it works for all states above a cut-off efficiency level $(\bar{m}, \bar{k}), \bar{m}<N-1, \bar{k}$ $\in 1, \ldots M$. Consider a state $(m, k)$ that is one level less efficient than $(\bar{m}, \bar{k})$. A contradiction to the theorem's claim would yield $\theta^{\prime}>0$, and a sequence $\Gamma_{p} \uparrow \infty$ with the property that for every $\Gamma_{p}$ there is a game $\left\langle T_{p}, \Phi, G\right\rangle, T_{p}$ $\geq \Gamma_{p}$, an initial $(m, k)$ state $a_{0}$, (a mixed strategy) equilibrium within the game $\alpha^{*}\left(. ; T_{p}\right)$ (with that initial state) and a time-period $t_{p} \geq \Gamma_{p}$ for which $\operatorname{Pr} o b .\left(\alpha_{t_{p}}^{*} \neq \widehat{a}\right)>\theta^{\prime}$.

By virtue of Lemma 3 , if $\alpha_{t_{p}}^{*} \neq \widehat{a}$ it follows that on that history $\alpha_{t}^{*} \neq \widehat{a}$, for all $t<t_{p}$. Hence, the payoffs of the mover in period 1 , say player $k$, over the first $t_{p}$ periods are bounded above by

$$
t_{p}\left[\theta^{\prime} \bar{\pi}_{k}+\left(1-\theta^{\prime}\right) \pi_{k}(\widehat{a})\right]
$$

where $\bar{\pi}_{k}$, as always, is the second-highest payoff for player $k$ in the stage game. By a logic identical to that of the previous proof, within at most $M$ periods we have to arrive at a state that can be raised in efficiency by the moving player. By virtue of the induction hypothesis, the period zero payoff to keeping alive the possibility of a switch is at least

$$
(1-\theta)\left[t_{p}-M-\Gamma_{\bar{m}, \bar{k}}(\theta)-1\right] \times \pi_{k}(\widehat{a})+\left[\theta\left(t_{p}-M-\Gamma_{\bar{m}, \bar{k}}(\theta)-1\right)+\left(M+\Gamma_{\bar{m}, \bar{k}}+1\right)\right] \times \underline{\pi}_{k}
$$

where $\underline{\pi}_{k}$ is the lowest payoff for player $k$ in the stage game. Furthermore, $\theta$ is chosen to be strictly smaller than $\theta^{\prime}$ and indeed such that $\left[\left(\theta^{\prime}-\theta\right)\left(\pi_{k}(\widehat{a})-\right.\right.$ $\left.\left.\bar{\pi}_{k}\right)-\theta\left(\bar{\pi}_{k}-\underline{\pi}_{k}\right)\right]>0$. The last expression is easily reduced to

$$
t_{p}\left[\theta \underline{\pi}_{k}+(1-\theta) \pi_{k}(\widehat{a})\right]-\left(M+\Gamma_{\bar{m}, \bar{k}}+1\right)(1-\theta)\left[\pi_{k}(\widehat{a})-\underline{\pi}_{k}\right]
$$

By grouping similar terms, from Eqs. 14 and 15 it can be shown that keeping the switch alive is more profitable if

$t_{p}\left\{\left(\theta^{\prime}-\theta\right)\left[\pi_{k}(\widehat{a})-\bar{\pi}_{k}\right]-\theta\left(\bar{\pi}_{k}-\underline{\pi}_{k}\right)\right\}>\left(M+\Gamma_{\bar{m}, \bar{k}}+1\right)(1-\theta)\left(\pi_{k}(\widehat{a})-\underline{\pi}_{k}\right)$

and that inequality must hold if $t_{p}$ is chosen appropriately large. The theorem is proved. $\diamond$

\subsection{Proof of Most General Theorem}

The same efficiency index that was defined for Model 2 will be used here. That the theorem holds for $N-1$ efficient states is obvious. The induction 
proof will closely parallel that of Lemma 6 with the only real departure being Case 3 in that proof. Hence that is the only case that will be dealt with in any detail here.

Lemma 12 For every $(m, k): m=0, \ldots N-2, k=1, \ldots N$ there is a number $\Gamma_{m, k}<\infty$ such that for any pure strategy subgame perfect equilibrium and in any subgame that starts from an $(m, k)$ efficient state, full efficiency is achieved within at most $\Gamma_{m, k}$ periods, i.e., $\alpha_{t}^{*}=\widehat{a} \forall t \geq \Gamma_{m, k}$.

Proof: Suppose, to begin with, that there is at least one mover in the current period. Suppose that the lemma holds for efficiency levels above a cut-off, say $(\bar{m}, \bar{k})$. Consider now a state $(m, k)$ that is less efficient than $(\bar{m}, \bar{k})$ by one order. Again, without loss, assume that one of the current movers is player 1 . There are three cases:

Case $1-m=\bar{m}-1$ (which means $k=1$ ). The proof is identical to that of Case 1 in Lemma 6.

Case $2-m=\bar{m}, 1<k<N$ (and, of course, $k=\bar{k}+1$ ). The proof is identical to that of Case 2 in Lemma 6.

Case $3-m=\bar{m}, k=N$ In this case the set of committed dates and the set of committed dates with efficient commitments are identical, i.e., $\Theta^{c}=\widehat{\Theta}$ and both sets might, of course, be empty.

Case $3 \mathrm{a}$ - If the sets are empty, i.e., all players have a move in the current period, $m=0$, and any current mover can increase efficiency by picking the efficient action and commiting to move again in period $t+N$.

Case $3 \mathrm{~b}$ - Suppose the sets are non-empty and consider first the case where there are multiple movers in the current period - one of them being, say, player 1 - and no mover in period $t+1$. Player 1 can always pick a date that is distinct from that of the other players and can pick the efficient action. If at least one of the other current movers commits to a period beyond $t+1$ then efficiency increases. ${ }^{30}$. Even if all of the other current movers choose to move again in period $t+1$ then again efficiency increases. ${ }^{31}$

Case $3 \mathrm{c}$ - Same as $3 \mathrm{~b}$ except there are mover(s) in date $t+1$. Player 1 's unilateral action then cannot increase efficiency if all the other movers at date $t$ decide to move again at date $t+1 .^{32}$ At date $t+1$, a mover

\footnotetext{
${ }^{30}$ If one of these other movers also picks the efficient action to commit to, then efficiency increases to at least $m+1$. If the commitments are all to inefficient actions, then $k$ becomes finite and consequently, again, efficiency increases.

${ }^{31}$ Since there are not any efficient commitments that are to expire in date $t+1$.

${ }^{32}$ Remember there is an efficient commitment that is expiring at date $t+1$ so player 1 's efficient commitment merely replaces that efficiency index loss.
} 
at that date - say, player 2 - can mimic what player 1 does at date $t$ (i.e., can commit to the efficient action till date $t+N+1$ ). If there is no precommitted mover at date $t+2$ we are in the world of Case $3 \mathrm{~b}$. Else, we are back in Case $3 \mathrm{c}$ and Player 2 is only able to maintain efficiency if all the other movers at $t+1$ choose to move again at date $t+2$. In other words then we will repeat Case $3 \mathrm{c}$ but at date $t+2$. Strictly before date $t+N-1$ we have to arrive at Case $3 \mathrm{~b}$, i.e., a state where are no precommitted movers in the subsequent period. A mover at that point can unilaterally increase efficiency by the above argument.

Case $3 \mathrm{~d}$ - If, on the other hand, there is a single mover in the current period that mover can maintain efficiency by picking the efficient action and commiting to move again in period $t+N$. Within $N-1$ periods, there has to be multiple movers. Then we are in case $3 \mathrm{~b}$ or $3 \mathrm{c}$.

So in all cases a mover can immediately - or within $N-1$ periods - strictly increase the efficiency index. By the induction hypothesis she can therefore implement full efficiency within at most $\Gamma_{\bar{m}}, \bar{k}$ periods. The argument of Case 1 then says that she will pass on doing so only if she believes that she can free-ride within a uniformly bounded number of periods anyway.

Suppose, finally, that there is no mover in the current period. By definition, within $N-1$ periods, there will be. The above argument can then be applied.

Hence the lemma is proved and so is the theorem. $\diamond$

\section{References}

Admati, A., and M. Perry, 1991, Joint Projects without Commitment, Review of Economic Studies 58, 259-276.

Anderlini, L., 1991, Communication, Computability, and Common Interest Games, mimeo, Cambridge University.

Abreu, D., P. K. Dutta and L. Smith, 1994, The Folk Theorem for Repeated Games: A NEU Condition, Econometrica 62, 939-948.

Aumann, R. and S. Sorin, 1989, Cooperation and Bounded Recall, Games and Economic Behavior 1, 5-39.

Benoit, J-P and V. Krishna, 1985, Finitely Repeated Games, Econometrica 53, 905-922.

Bhaskar, V. and F. Vega-Redondo, 1998, Asynchronous Choice and Markov Equilibria: Theoretical Foundations and Applications, mimeo, University of Essex. 
Cooper, R., 1999, Coordination Games: Complementarities and Macroeconomics, Cambridge University Press, Cambridge, UK.

Cooper, R., and A. John, 1988, Coordinating Coordination Failures in a Keynesian Model, Quarterly Journal of Economics 103, 441-464.

Cooper, R., D. V. DeJong, R. Forsythe, and T. W. Ross, 1990, Selection Criteria in Coordination Games, American Economic Review, 80, 218-233.

Crawford, V., 1995, Adaptive Dynamics in Coordination Games, Econometrica 63, 103-143.

Cyert, R., and M. DeGroot, 1970, Multiperiod Decision Models with Alternating Choice as a Solution to the Duopoly Problem, Quarterly Journal of Economics 84, 410-429.

Dutta, P. K., 1995, A Folk Theorem for Stochastic Games, Journal of Economic Theory 66, 1-32.

Eaton, J., and M. Engers, 1990, Intertemporal Price Competition, Econometrica 58, 637-659.

Fudenberg, D., and E. Maskin, 1986, The Folk Theorem in Repeated Games with Discounting and Incomplete Information, Econometrica, 54, 533-554.

Gale, D. 1995, Dynamic Coordination Games, Economic Theory 5, 1-18.

Gale, D., 2000, Monotone Games with Positive Spillovers, mimeo, New York University.

Lagunoff, R. and A. Matsui, 1997, Asynchronous Choice in Repeated Coordination Games, Econometrica 65, 1467-1478.

Lagunoff, R. and A. Matsui, 2002, Are Anti-Folk Theorems in Repeated Games Non-Generic, Review of Economic Dynamics, 6, 397-414.

Maskin, E. and J. Tirole, 1988a, A Theory of Dynamic Oligopoly, I: Overview and Quantity Competition with Large Fixed Costs, Econometrica $56,549-570$.

Maskin, E. and J. Tirole, 1988b, A Theory of Dynamic Oligopoly, II: Price Competition, Kinked Demand Curves and Fixed Costs, Econometrica $56,571-600$.

Matsui, A., 1991, Cheap Talk and Cooperation in the Society, Journal of Economic Theory 54, 245-258.

Tirole, J., 1989, The Theory of Industrial Organization, MIT Press, Cambridge, Mass.

VanHuyck, J., R. C. Battalio, and R. O. Beil, 1990, Tacit Coordination Games, Strategic Uncertainty and Coordination Failure, American Economic Review, 80, 234-248. 
Wallner, K., 1999, Sequential Moves and Tacit Collusion: Reaction Function Cycles in a Finite Pricing Duopoly, Journal of Economic Theory, 84, 255-261.

Wen, Q., 1998, A Folk Theorem in Repeated Sequential Games, mimeo, University of Windsor. 